\title{
4D Shape-Preserving Modelling of Bone Growth $^{\star}$
}

\author{
Per Rønsholt Andresen ${ }^{1,2}$, Mads Nielsen², and Sven Kreiborg ${ }^{2}$ \\ 1 Department of Mathematical Modelling, \\ Technical University of Denmark, Denmark \\ pra@imm.dtu.dk http://www.imm.dtu.dk/ pra \\ 2 3D-Lab, School of Dentistry, University of Copenhagen, Denmark \\ http: //www. lab3d.odont.ku.dk
}

\begin{abstract}
From a set of temporally separated scannings of the same anatomical structure we wish to identify and analyze the growth in terms of a metamorphosis. That is, we study the temporal change of shape which may provide an understanding of the biological processes which govern the growth process. We subdivide the growth analysis into growth simulation, growth modelling, and finally the growth analysis. In this paper, we present results of growth simulation of the mandible from 3 scannings of the same patient in the age of 9 months, 21 months, and 7 years. We also present the first growth models and growth analyzes. The ultimative goal is to predict/simulate human growth which would be extremely useful in many surgical procedures.
\end{abstract}

\section{Introduction}

This paper presents a non-linear growth model which to a very good approximation interpolates the growth as seen on the human mandible (the lower jaw). The results comply with the existing $2 \mathrm{D}$ theory on mandibular growth [1]. These experiments use a unique $4 \mathrm{D}$ data set containing three Computerized Tomography (CT) scans ${ }^{1}$ of the same patient with Apert syndrom, but with normal mandibular development, taken at three ages (9 months, 21 months, and 7 years old). In many situations, surgeons need information about the growth of the jaws, particularly when performing pediatric cranio-facial surgery. After surgery, the bones continue to grow, and therefore in order to optimize the intervention, there is a need to predict/simulate growth. Also for basic understanding and teaching, we have a need for these models. We subdivide the growth study into growth simulation, growth modelling, and finally the growth analysis. Growth simulation is the data driven analysis, where we try to fit an (almost) arbitrary model to the data. In growth modelling, we have a model and wish to evaluate if the data fits the model. When we are doing growth analysis, the process is

\footnotetext{
* This work is partly supported by the Danish Technical Research Council, registration number 9600452

1 The scans were performed for diagnostic and treament planning purposes.
} 
reversed, and we try to extract information from the models, such as active areas, spatial correlations, predicted changes, etc. In contrast to normal biological tissue growth, bone grows only on the surface. The interior is rigid and does not change shape [1]. The growth of a bone can be subdivided into deposition (adding bone) and resorption (removal of bone). Because the deposition and resorption happen all over the surface of the bone at different speeds, this results in non-linear growth [1]. For the mandible the condyles are the most active areas, and are therefore important to be followed over time. Homologous ${ }^{2}$ points followed over time, define a spatio-temporal vector field (the growth vector field or just vector field or flow field). The goal of growth simulation is the identification of the spatio-temporal vector field. Many different vector fields will satisfy the constraints given by the data and the definitions of homologous points. Thus a growth model (or interpolation model) must be used for the determination of a unique vector field.

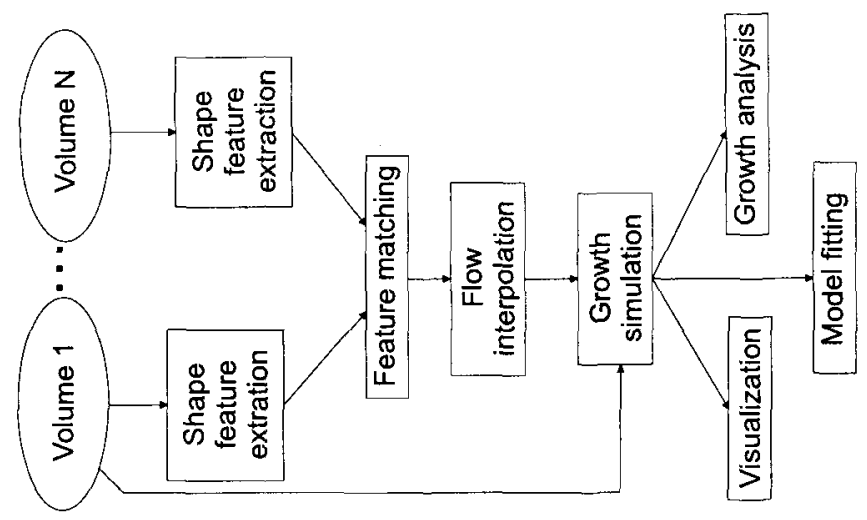

Fig. 1. Flow chart of the algorithms involved in the growth analysis.

We distinguish between models having the same number of degrees of freedom as the data and over-constrained models. We will use the first in the process of growth simulations, while over-constrained models are used for growth modelling. The simulation is a mere data interpolation, whereas the modelling will test whether data comply to a given model. In Figure 1 the information flow is shown.

Finally, in the growth analysis, we will extract information from the simulated or modelled vector field in order to identify local biological processes and/or physical conditions that govern the remodelling of the bone. In this paper, we estimate the resorption and deposition on the surface of the mandibular bone.

In earlier work on simulating the growth of the mandibular bone [2] the interpolation has been performed directly on the surface position. But the time steps are large, and a direct surface position interpolation as carried out in

$\overline{{ }^{2} \text { Homologous }}=$ having the same relative position, value, or structure. 
that work will not preserve the overall shape. Thus, intermediate steps will not necessarily look like mandibles. See figure 2 .
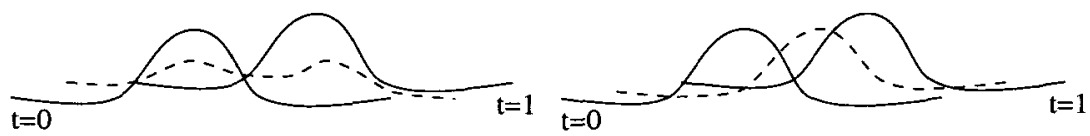

Fig. 2. Surface interpolation illustration to the left is a linear interpolation in position of closest point. The top at $t=0$ will disappear and at the same time a new top will appear. To the right is a linear interpolation of shape feature positions such as maximally curved points on the surface. Here, the top moves to the right over time.

In Section 2, we will give one definition of homologous points in terms of the extremal mesh [15] (which are lines) and the different types of ridge lines. These homologous equivalent lines are matched, as described in Section 3. Since this yields a very sparse vector field the interpolation becomes crucial and is described in Section 4. In Section 5, we describe existing 2D models of the growth of the mandible, and use these models for a 3D growth modelling. In Section 6, we extract properties of the modelled flow fields such as the local amount of resorption and deposition. Section 7 discusses our results and describes future work.

\section{Local Shape Features}

The growth vector field links homologous points, or points of equivalent morphology. In this section, we define equivalence classes of points on a surface. The local shape of a surface is totally characterized by the principal curvatures $k_{1}, k_{2}\left(k_{1}>k_{2}\right)$ and their derivatives in the coordinate system defined by the principal directions $\left(t_{1}, t_{2}\right)[6]$. Since the bone topology is not changing in our
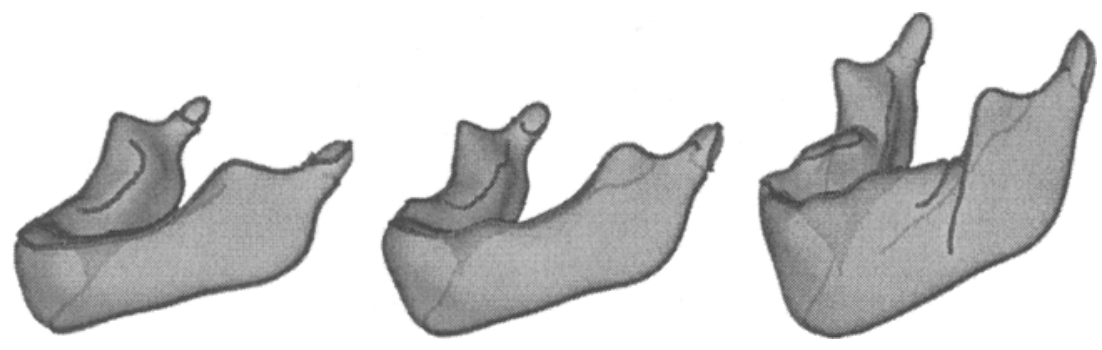

Fig. 3. The crest lines on the three smoothed mandibles at 9 months (left), 21 months (middle), and 7 years old (right). The surfaces are translucent.

studies, we may model the growth process by a 3D diffeomorphism (a one-to-one 
differential mapping). This corresponds to D'Arcy Thompson classical methods of transformations [5]. The principal curvatures and directions will in general change when exposed to this non-linear diffeomorphism, and cannot directly be used for registration. However, certain shape singularities are stable in the sense that they cannot be removed by an infinitesimal perturbation [3]. Here, we give a list of some stable shape features.

\begin{tabular}{lcc} 
Shape feature & Definition & Dimensionality \\
\hline Umbilic point & $k_{1}=k_{2}$ & 0 \\
Critical curvedness [11] & $\partial_{t_{1}} C=0 \wedge \partial_{t_{2}} C=0$, def: $C=k_{1}^{2}+k_{2}^{2}$ & 0 \\
Extremal points & $\partial_{t_{1}} k_{1}=0 \wedge \partial_{t_{2}} k_{2}=0$ & 0 \\
Parabolic line & $k_{1}=0 \vee k_{2}=0$ & 1 \\
Ridge line (or extremal mesh) & $\partial_{t_{1}} k_{1}=0 \vee \partial_{t_{2}} k_{2}=0$ & 1 \\
Crest line & $\partial_{t_{1}} k_{1}=0 \wedge \partial_{t_{1}^{2}} k_{1}<0$, def: $\left|k_{1}\right|>\left|k_{2}\right|$ & 1 \\
Sub-parabolic line [3] & $\partial_{t_{2}} k_{1}=0 \vee \partial_{t_{1}} k_{2}=0$ & 1
\end{tabular}

Shape features with dimension $\geq 2$ will not be discussed in this paper.

The ridge lines (or extremal mesh) can be partitioned into four types corresponding to respectively maximum or minimum in $k_{1}$ and $k_{2}$. We use the maxima in (the absolute value of) both $k_{1}$ and $k_{2}$.

The above mentioned shape features are all structurally stable, but even though they can not be removed by infinitesimal perturbations, they will in general change topology under finite perturbations.

We work with the extraction and matching of ridge lines in a scale-space setting [10] (see the following section). Also the scale-space evolution of ridge lines is not totally understod even though some aspects are covered in the literature $[4,7,8]$. Thus, theoretical issues are still to be clarified. However, by making a matching which only accepts good matches (see the following section), we obtain satisfying results. The crest lines of the mandibles can be seen in Figure 3.

\section{$3 \quad$ Feature Matching}

As features we will only consider the lines with maximally $k_{1}$ (crest-lines) and maximally $k_{2}$ (here, called $k_{2}$-max lines) in the extremal mesh. The overall framework follows the ideas of [14]. First we extract the crest lines and $k_{2}$-max lines for each dataset at scale 3.0 (matching scale) and 1.0 (localization scale). The crest lines at scale 3.0 are registered pairwise (here, it means only the temporally neighboring datavolumes), and initial vector fields are calculated. The $k_{2}$-max lines are then deformed according the initial vector fields and registered. From the two sets of matches (one from the crest-lines, the other from the $k_{2}$-max lines) final vector fields are calculated. This procedure is repeated for scale 1.0, but the lines are initially deformed according to the the final vector fields for scale 3.0 .

The steps in the registration are always the same. First moment-registration, then two first order polynomial deformations, followed by two second order polynomial deformations. Lastly a totally non-rigid deformation is applied (all points 
on the lines move freely). For all the registration methods (including the nonrigid) they must satisfy the restriction that the deformation must be a $3 \mathrm{D}$ diffeomorphism. See Figure 6 for an example of matches between two set of crest lines at scale 3.0 .

\section{Flow Interpolation}

The matching provides us with a very sparse set of vectors. This vector field must be interpolated such as to yield a differentiable spatially dense field of spatiotemporal deformation vectors: A diffeomorphism (that its, spatial the Jacobian is nowhere vanishing).

We wish the interpolation to satisfy the following constraints: (i) approximation, (ii) regularity, (iii) shadowing, (iv) maximum principle. (i) The interpolated vector field must approximate the data values well since localization of the features are assumed relatively precise. (ii) In regions of missing features a smooth solution must be created. We do assume a regular growth. (iii) The data must be able of shadowing each other. That is, in a given direction only the nearest data must be weighted. In this way, we avoid that features from the "other side" of a thin structure influence the local solution. (iv) The solution must not extend the solution to values larger than the largest data value or smaller than the smallest data value. We assume that the ridge lines also correspond to lines of extreme growth.

We address this as a statistical inference problem. Assume that the covariance function $C\left(x, x^{\prime}\right)$ is known. The covariance function expresses the covariance of the vector field values in two points $x$ and $x^{\prime}$. Typically, the closer the points are, the more correlated their data values are assumed to be. An interesting aspect is that if this covariance defines a distribution of functions, and if $C\left(x, x^{\prime}\right)=$ $\exp \left(-\left(\left|x-x^{\prime}\right| / \lambda\right)^{\alpha}\right)$, some well-known function classes appear with probability 1, for different choices of $\alpha: \alpha=0$ yields white noise, $\alpha \in] 0 ; 2$ [ yields fractional Brownian motions with $\alpha=1$ as the classical Brownian motion [12], while $\alpha=2$ (the Gaussian) yields $C^{\infty}$ functions. Given the covariance function $C\left(x, x^{\prime}\right)$ and an expression of the belief in data as the assumed variance of data values $r^{2}$, we can make a maximum likelihood estimation of $f(x)$ as [16]

$$
f(x)=\frac{w(x, \boldsymbol{x}) Q^{-1} g(\boldsymbol{x})}{w(x, \boldsymbol{x}) Q^{-1} \mathbf{1}}
$$

where $w(x, \boldsymbol{x})$ is a vector containing $w_{i}=C\left(x, x_{i}\right)$, and $Q$ is a matrix containing $Q_{i j}=C\left(x_{1}, x_{2}\right)+r^{2} \delta_{i j}$. The intuitive interpretation of the introduction of $Q^{-1}$ is that, prior to the regularizations based on the covariance function, an inverse filtering is performed to make the samples uncorrelated. In terms of scale-space, we might say that we have data given at some scale $\lambda$. To interpolate, we first perform a deblurring to scale zero, then interpolate and then blur back to the current scale.

This method satisfies all criteria when $\alpha=1$ and $r=0$ [13]. $\lambda$ can be chosen freely, so as to adjust the smoothness of the interpolated vector field. In 
Figure 4, the deformation of the mandible is shown as it is transported along the deformation vector field.
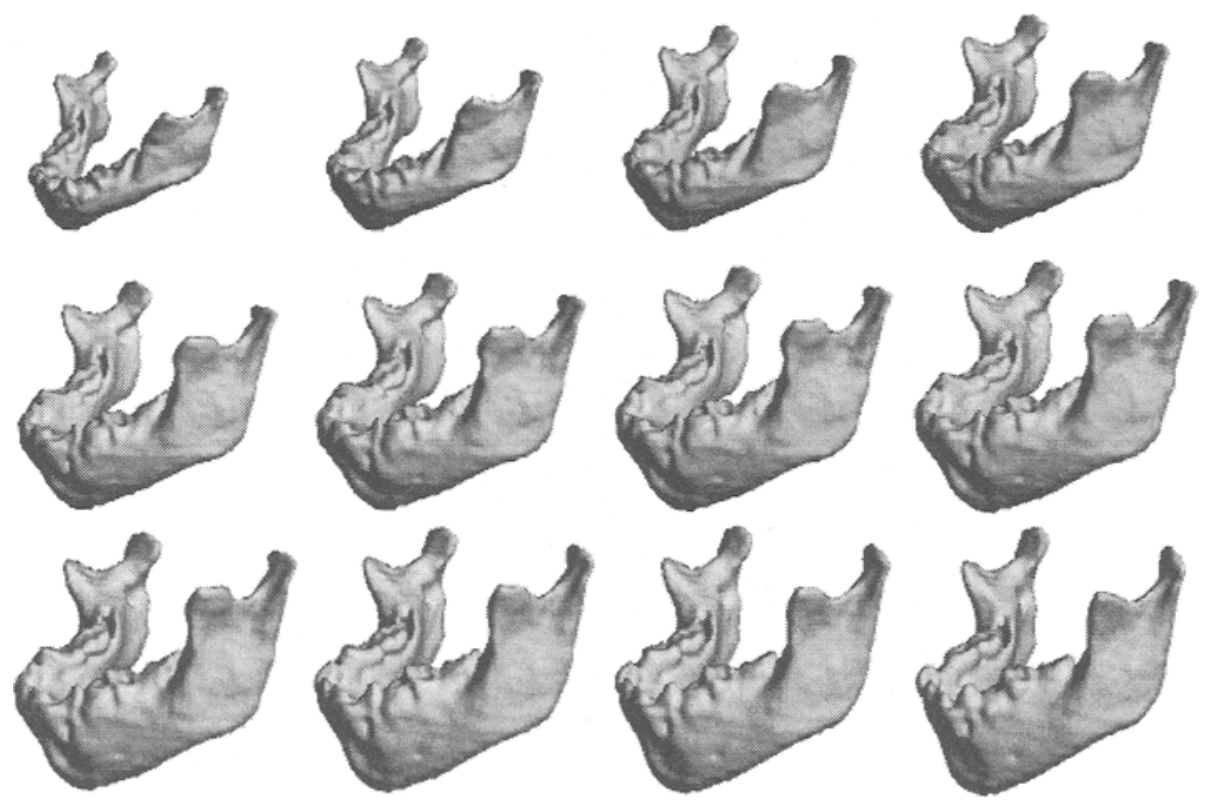

Fig.4. Result of deformations on the 7 years mandible using a second order polynomial model (see section 5). The top left and right images are the deformation at 9 months and 21 months, respectively. The bottom right image is the original 7 years old mandible.

\section{Growth Modelling}

We have the general model $\boldsymbol{g}(\boldsymbol{\theta}(x, y, z), t), \boldsymbol{g}: \mathcal{R}^{3} \mapsto \mathcal{R}^{3}$ (for fixed t), and the 3D volumes $v_{i}(x, y, z)$, where $\theta(x, y, z)$ is the parameters for $g$, and $(x, y, z)$ defines a point in $\mathcal{R}^{3} . t$ is the time. $i=\{1, \ldots, n\} . n$ is the total number of volumes. $t_{i}$ is the time at the $i$ th scan. We need to pick a reference volume, let's say $v_{n}$. All deformations will then be applied to this set, i.e. a simulated volume at time $t$ is given by $\tilde{v}_{n}\left(\boldsymbol{g}(\theta(x, y, z), t), v_{n}(x, y, z)\right)$ or $\tilde{v}_{n}(x, y, z, t)$ for short. We want to solve the problem

$$
\hat{\theta}=\arg \min _{\theta} \sum_{i=1}^{n}\left[\sum_{x, y, z}\left\{\tilde{v}_{n}\left(x, y, z, t_{i}\right)-v_{i}\left(x, y, z, t_{i}\right)\right\}^{2}\right]
$$

Note, when having $\boldsymbol{g}(\boldsymbol{\theta}(x, y, z), t)$, the actual deformation on the volume $v_{n}$ from time $t_{n}$ to $t$, can always be made by a linear deformation (we just pick the straight 

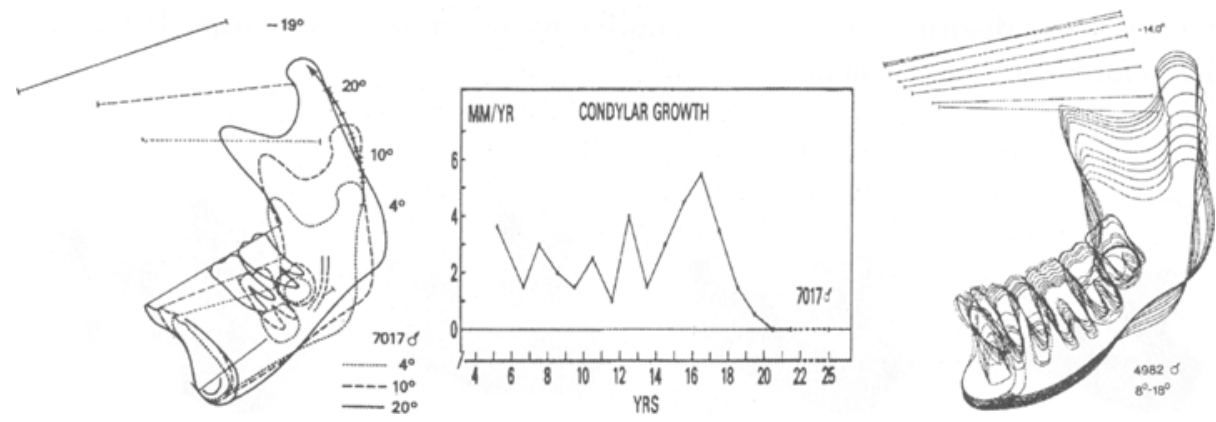

Fig. 5. Left: Mandibular tracing at three age stages (this is not the same patient as for the CT scans) superimposed in a reference line in the corpus with reference to natural structures. Middle: Curve for yearly rate of condylar growth. Both plots are data from the same patient with a normal mandibular growth. Right: Mandibular growth tracing superimposed by means of metallic implants, illustrating the yearly growth and remodelling of the mandible and the eruption if the teeth, as seen in profile view. From [1].

line between two homologous points in $\tilde{v}_{n}(x, y, z, t)$ and $\left.v_{n}(x, y, z)\right)$. In general, this leads to a non-linear optimization problem, but if we pick models, linear in the parameters, regression analysis [9] can be used. Linear models

$$
\boldsymbol{g}(\theta(x, y, z), t)=\theta(x, y, z) * t
$$

have been used in previous work [2]. This model has the drawback that a point, $\boldsymbol{p}$, can only grow in the direction of the vector $\theta(p)$. From Figure 5 , it is obvious that the growth of the mandible is not linear. The simplest non-linear model is a polynomial model (with $k \leq 2$ )

$$
g(\theta(x, y, z), t)=t^{k} \theta_{k}+\cdots+t^{2} \theta_{2}+t \theta_{1}+\theta_{0}, \boldsymbol{\theta}=\left[\theta_{k} \cdots \theta_{2} \theta_{1} \theta_{0}\right]
$$

As seen from Figure 5, the growth speed is not constant, but this can be handled by the model by re-parametrizing the time variable, $t$.

Because we only have three scans of the same patient, we can not go above the second order model ${ }^{3}(\mathrm{k}=2)$. A second order polynomial model is estimated using the matches between scan one and two, and scan two and three. Interpolation of the volumes is carried out by deforming the last scan (see Figure 4). Because the calculation of the deformation field from one scan to the next scan is not perfect, we have some model errors (even though the model itself doesn't have any error) which are seen in Figure 7. Other possible models include logarithmic spirals and power functions, known from the theory of growth [5] or spatially constrained models.

\footnotetext{
${ }^{3}$ This leads to a model error equal zero, because the number of parameters equals the number of volumes.
} 


\section{Growth Analysis}

The growth modelling is on its own also a growth analysis since residuals to an over-constrained model may be used for validating the model. The growth simulations, as we obtain it in Figure 4, can be used for a local characterization of the growth. The model errors at 9 months are shown in Figure 7. Using anatomical structures which are also spatially stable, a rigid registration of the different time instances of the bone can be obtained. In the mandible, the nerve canal is known to be spatially stable, and can serve as an anchor for a rigid registration. In this coordinate system, the spatio-temporal growth simulation vector field can be used directly for estimation of the amount of surface resorption and deposition. In Figure 8 we show the surface remodelling in terms of a color coding of the mandible as respectively the remodelling (the local velocity vector projected to the surface normal) and the speed of the homologous points. The remodelling is consistent with earlier 2D studies on larger statistical material [1]. Especially we see the expected large movement of the condyle.

\section{Summary}

We have simulated the growth of the mandible from 3 CT scans of the same patient at ages 9 months, 21 months, and 7 years. The intermediate interpolated time instances also exhibit shapes that clearly are "mandible shaped". This is due to the strategy of interpolating in shape feature position instead of a simple surface position interpolation [2]. The major errors in the simulations are found in the region where teeth are appearing. In principle, they should a priori have been removed from the mandible surfaces, as they are not part of the mandible but separate objects, and the shape change can not be contributed to a surface remodelling. The shape modelling in this paper has used simple second order polynomial temporal models. They exhibit some inexpedient features inherent for polynomial approximations. An example is a tendency to a contraction of the two condyles towards each other if a time extrapolation is attempted. Since the ultimate goal of a growth analysis and modelling is a prediction of the shape of the craniofacial complex. Future work will be devoted to examination of superior temporal models and validation on more datasets. Extension of the feature matching from ridge lines to iso-surfaces, as mentioned in Figure 7, may reduce errors. Also development of a skeletal growth atlas, which contains growth models for all bones would be interesting.

\section{References}

1. A. Björk and V. Skieller. Normal and abnormal growth of the mandible. A synthesis of longitudinal cephalometric implant studies over a period of 25 years. European Journal of Orthodontics, 5:1-46, 1983.

2. M. Bro-Nielsen, C. Gramkow, and S. Kreiborg. Non-rigid image registration using bone growth model. In CVRMed-MRCAS'97, Lecture Notes in Computer Science, pages 3-12. Springer Verlag, 1997. 
3. J. W. Bruce and P. J. Giblin. Curves and Singularities. Cambridge University Press, Cambridge, 1984.

4. J. Damon. Local Morse theory for solutions to the heat equation and Gaussian blurring. Jour. Diff. Eqtns., 1993.

5. D'Arcy Thomsen. On Growth and Form. Cambridge Unversity Press, 1971.

6. M. P. Do Carmo. Differential Geometry of Curves and Surfaces. Prentice Hall, Inc., New Jersey, 1976.

7. D. Eberly, R. Gardner, B. Morse, S. Pizer, and C. Scharlach. Ridges for image analysis. Journal of Mathematical Imaging and Vision, 4(4):353-373, 1994.

8. M. Fidrich. Following feature lines across scale. In ScaleSpace97, 1997.

9. D. Kazakos and P. Papantoni-Kazakos. Detection and Estimation. Computer Science Press, W. H. Freemann and Company, New York, 1990.

10. J. J. Koenderink. The structure of images. Biological Cybernetics, 50:363-370, 1984.

11. J. J. Koenderink. Solid Shape. MIT Press, Cambridge, Massachusetts, 1990.

12. B. B. Mandelbrot and J. W. van Ness. Fractional Brownian motions, fractional noises, and applications. SIAM Review, 10(4):422-437, 1968.

13. M. Nielsen and P. R. Andresen. Feature displacement interpolation. In IEEE 1998 International Conference on Image Processing, Chicago, Illinois, USA, 1998. To appear.

14. G. Subsol, J.-P. Thirion, and N. Ayache. A general scheme for automatically building 3D morphometric anatomical atlases: application to a skull atlas. Medical Image Analysis, 2(1):37-60, 1998.

15. J.-P. Thirion. The extremal mesh and the understanding of 3D surfaces. International Journal of Computer Vision, 6(58):503-509, November 1996.

16. C. K. I. Williams and C. E. Rasmussen. Gaussian processes for regression. Advances in Neural Information Processing Systems, 8, 1996.

Comments on color images. Fig. 6: The final matches (lines in black) between two sets of crest lines. The crest lines on the 21 months and 7 years mandible are red and green, respectively. It is seen that the condyles on the two mandibles are matched together. For visual clarity only every eighth match is shown. Fig. 7: The plots in the middle and right shows the frequency and accumulated distribution of the distance errors (the distance errors are measured as the minimal distances from the deformed surface to the original surface) between the 9 months old mandible and the 7 years old mandible deformed to 9 months. The mean error is $0.57 \mathrm{~mm}$, and $95 \%$ of the errors are less than $1.46 \mathrm{~mm}$. The maximal error is $2.79 \mathrm{~mm}$. This should be compared to the size of the 7 years old mandible whice is approximately $(X, Y, Z)=(80 \mathrm{~mm}, 100 \mathrm{~mm}, 40 \mathrm{~mm})$. The left surface is colored red when the error $>1.46 \mathrm{~mm}$, else white. When the surface changes a lot the matching algorithm does not match with lines in the "holes" of the surface, but are more likely to match with a line on the "top", therefor we see the errors located at places with a lot of changes in the shape. If we applied a surface to surface registration afterwards, the errors would be minimal. Fig. 8: First row: The 7 years mandible colored with the local velocity vector projected to the surface normal (left) and the length of the velocity vector (right). The next row shows $\mathrm{X}$ (first two images), $\mathrm{Y}$ (next two images), and Z (last two images) components of the velocity vector (projection and length, respectively). Read text in "Growth Analysis" for further explanation. 


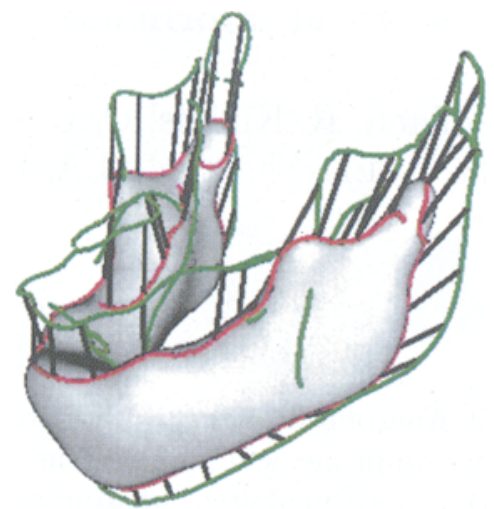

Fig. 6. See "Comments on color images".
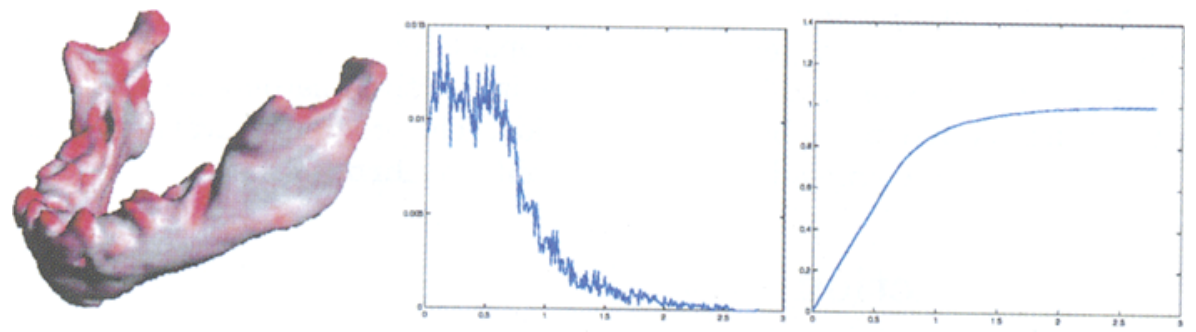

Fig. 7. See "Comments on color images".
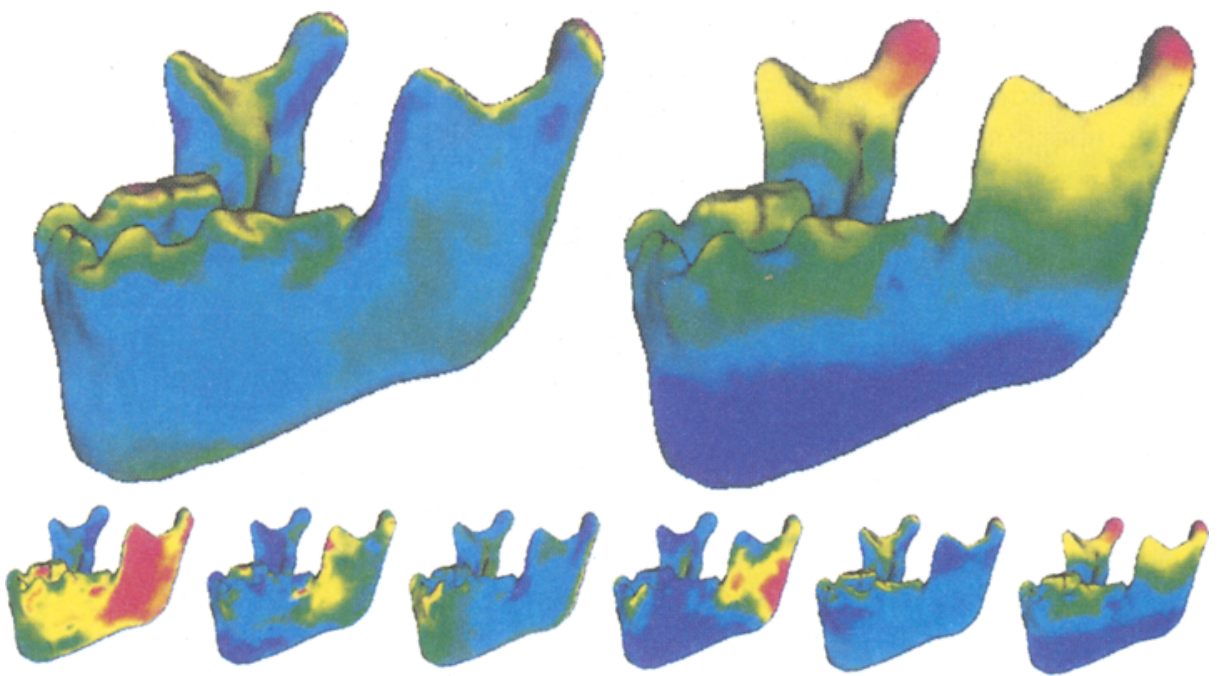

Fig. 8. See "Comments on color images". 